Review Article

\title{
Prescription of Chinese Herbal Medicine in Pattern-Based Traditional Chinese Medicine Treatment for Depression: A Systematic Review
}

\author{
Wing-Fai Yeung, ${ }^{1}$ Ka-Fai Chung, ${ }^{2}$ Ka-Yan $\mathrm{Ng}^{2}{ }^{2}$ Yee-Man Yu, ${ }^{2}$ Shi-Ping Zhang, \\ Bacon Fung-Leung $\mathrm{Ng}^{4}$ and Eric Tat-Chi Ziea ${ }^{4}$ \\ ${ }^{1}$ School of Chinese Medicine, University of Hong Kong, Hong Kong \\ ${ }^{2}$ Department of Psychiatry, University of Hong Kong, Pokfulam Road, Hong Kong \\ ${ }^{3}$ School of Chinese Medicine, Hong Kong Baptist University, Hong Kong \\ ${ }^{4}$ The Chinese Medicine Department, Hospital Authority, Hong Kong \\ Correspondence should be addressed to Ka-Fai Chung; kfchung@hkucc.hku.hk
}

Received 16 April 2014; Accepted 21 September 2014

Academic Editor: Guo-Zheng Li

Copyright (C) 2015 Wing-Fai Yeung et al. This is an open access article distributed under the Creative Commons Attribution License, which permits unrestricted use, distribution, and reproduction in any medium, provided the original work is properly cited.

\begin{abstract}
Traditional Chinese medicine (TCM) treatments are often prescribed based on individuals' pattern diagnoses. A systematic review of randomized controlled trials in Chinese and English literatures on TCM pattern-based treatment for depression has therefore been conducted. A total of 61 studies, 2504 subjects, and 27 TCM patterns were included. Due to the large variation of TCM pattern among participants, we only analyzed the top four commonly studied TCM patterns: liver qi depression, liver depression and spleen deficiency, dual deficiency of the heart, and spleen and liver depression and qi stagnation. We found that Xiaoyao decoction was the most frequently used herbal formula for the treatment of liver qi depression and liver depression with spleen deficiency, while Chaihu Shugan decoction was often used for liver depression and qi stagnation. Bai Shao (Paeonia lactiflora Pall.) and Chai $\mathrm{Hu}$ (Bupleurum chinense DC.) were commonly used across different TCM patterns regardless of the prescribed Chinese herbal formulas. The rationale underlying herb selection was seldom provided. Due to the limited number of studies on TCM patternbased treatment of depression and their low methodological quality, we are unable to draw any conclusion regarding which herbal formulas have higher efficacy and which TCM patterns respond better to CHM.
\end{abstract}

\section{Introduction}

According to the Global Burden of Disease Study 2010, major depressive disorder (MDD) was ranked the second leading cause of years lived with disability, accounting for $8.2 \%$ of all years lived with disability [1]. The World Mental Health Survey Initiative showed that the average lifetime prevalence for major depressive episode based on the Diagnostic and Statistical Manual, Fourth Edition [2], was 14.6\% in 10 highincome countries and $11.1 \%$ in 8 low- to middle-income countries [3]. Pharmacotherapy is currently the most commonly used treatment for MDD because of its reported effectiveness; however, complaints such us nausea, headache, insomnia, agitation, weight gain, daytime somnolence, and sexual dysfunction are common during the course of treatment, leading to early termination in some patients. The use of psychotherapy as an alternative is no better because of its time-intensive nature, limited access to skilled providers, high cost, and requirement of patients' participation and motivation. Faced with the limitations of the currently available treatments, complementary and alternative medicine for depression is very common. A national representative survey in the United States found that $53.6 \%$ of people with depression reported using some forms of complementary and alternative therapies to treat depression during the past 12 months [4].

Chinese herbal medicine (CHM) is one of the oldest medical treatments in the world and it is a common form of complementary and alternative medicine therapy for MDD $[5,6]$. Previous studies have been conducted to examine the efficacy of CHM for depression [7-14]; however, limited 
information is available on pattern-based CHM treatment. According to the traditional Chinese medicine (TCM) theory, eight major parameters, yin and yang, external and internal, hot and cold, and excess and deficiency, are used to describe the patterns of bodily disharmony. Additional systems, such as qi, blood, and body fluid differentiation and zang $f u$ (organ) differentiation are also used [15]. In terms of the TCM theory, the onset of depression is often due to "damages" by extreme emotions. Liver qi is first affected, followed by disharmony of the $q i$ mechanism of the five viscera, particularly liver, spleen, and heart, resulting in a loss of regulation of the $q i$ and blood. The liver depression may repress the spleen and lead to consumption and damage of the heart qi. If heart loses its nourishment and the restfulness to heart shen (spirit) occurs, it will lead to unstable and depressed mood. When qi depression is prolonged, it will accumulate and transform into fire [16, 17].

TCM pattern differentiation is a diagnostic conclusion of the pathological changes of a disease state based on an individual's symptoms, physical signs, pulse form, and tongue appearance. Although it is believed that patternbased TCM treatment will provide better efficacy, previous studies regarding the additional benefits of TCM pattern differentiation are scarce. One randomized controlled trial (RCT) found that the therapeutic effect of Chinese herbal treatment according to TCM pattern was more sustainable than a standard formula in treating irritable bowel syndrome [18]. It has also been reported in rheumatoid arthritic patients that TCM pattern diagnoses can guide the use of Western medicine [19]. To the best of our knowledge, no systematic review has been conducted on pattern-based CHM treatment for depression. Given the high prevalence of depression and its frequent presentation to TCM practitioners, it is important to review the current application of pattern differentiation in CHM treatment for depression. The objectives of this paper were (1) to summarize the commonly diagnosed TCM patterns in patients with depression and (2) to find out the current practice of pattern-based CHM treatment for depression.

\section{Materials and Methods}

The present study was part of our systematic review on Chinese herbal medicine for depression [11, 20]. Two researchers (Ka-Yan $\mathrm{Ng}$ and Yee-Man Yu) independently searched nine Chinese language databases (China Journals Full-text Database, China Proceedings of Conference Full-text Database, Chinese Biomedical Literature Database, China Doctor Dissertations Full-text Database, China Master Theses Full-text Database, Chinese Science and Technology Documents Database, Chinese Dissertation Document Bibliography Database, Taiwan Electronic Periodical Services, and WanFang Database) and seven English language databases (MEDLINE, EMBASE, Cochrane Central Register of Controlled Trials, Cumulative Index to Nursing and Allied Health Literature, Allied and Complementary Medicine, PsycINFO, and ProQuest Dissertations and Theses A\&I) using the grouped terms "depression" or depressive* or dysthymia* or mood disorder* or "affective disorder*" or "affective symptoms" or MDD" and "Chinese herb* or herbal medicine* or traditional Chinese medicine* or TCM or ChaiHu-Shu-Gan-San or ChaiHuShuGan* or Xiao-Yao-San or

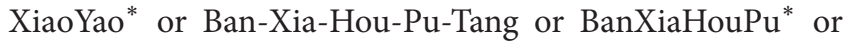
Gan-Mai-Da-Zao-Tang or GanMaiDaZao* or Gui-Pi-Tang or GuiPi* or Wen-Dan-Tang or WenDan or Yue-Ju-Wan or Yue-Ju" and the equivalent Chinese terms. We imposed no language restriction. We also checked the reference lists of the included papers and previous systematic reviews [7-14] for relevant articles.

2.1. Selection Criteria. Studies included in this review were RCTs that described TCM patterns of depressed subjects who received CHM treatment for depression. In order to obtain a full coverage of the topic, we had not set any specification for outcome measure and study quality. In addition, to derive a general picture of TCM pattern utilization, studies were excluded if they (1) had less than 30 subjects; (2) examined male or female only; (3) focused on subjects aged below 18 or above 70 years; (4) focused on a particular life transition period or a specific TCM pattern; (5) had no statistical information regarding the frequency of individual TCM pattern; or (6) were duplicated publications.

2.2. Data Extraction Process. Any disagreement about the eligibility of a study was resolved by discussion between the two researchers who independently selected the relevant publications and by consultation with the senior authors (Wing-Fai Yeung and Ka-Fai Chung). One author extracted the data (Ka-Yan Ng) and the other (Yee-Man Yu) checked the extracted data. For each study, the following variables were extracted: study design, sample size, mode of recruitment, sampling and diagnostic procedure, inclusion and exclusion criteria, and participants' characteristics including age, gender, and duration of depression. TCM patterns, treatment principles, treatment regimen and outcome of TCM treatments were obtained. All Chinese to English translations were deduced primarily from the World Health Organization (WHO) International Standard Terminologies on Traditional Medicine in the Western Pacific Region [21] and additionally from Traditional Chinese Internal Medicine [22], a commonly used English-language TCM textbook in China.

2.3. Study Quality Assessment. We assessed the methodological quality using the Jadad scale [23]. Points are awarded if the study is described as randomized, 1 point; has appropriate randomization method, 1 point; is described as double-blind, 1 point; uses appropriate blinding method, 1 point; or has description of withdrawals and dropouts, 1 point. A Jadad scale score $\geq 3$ represents better quality trials.

2.4. Statistical Analysis. SPSS version 20.0 was used for statistical analysis. Data were summarized using mean (SD) and $95 \%$ confidence intervals (CIs). 


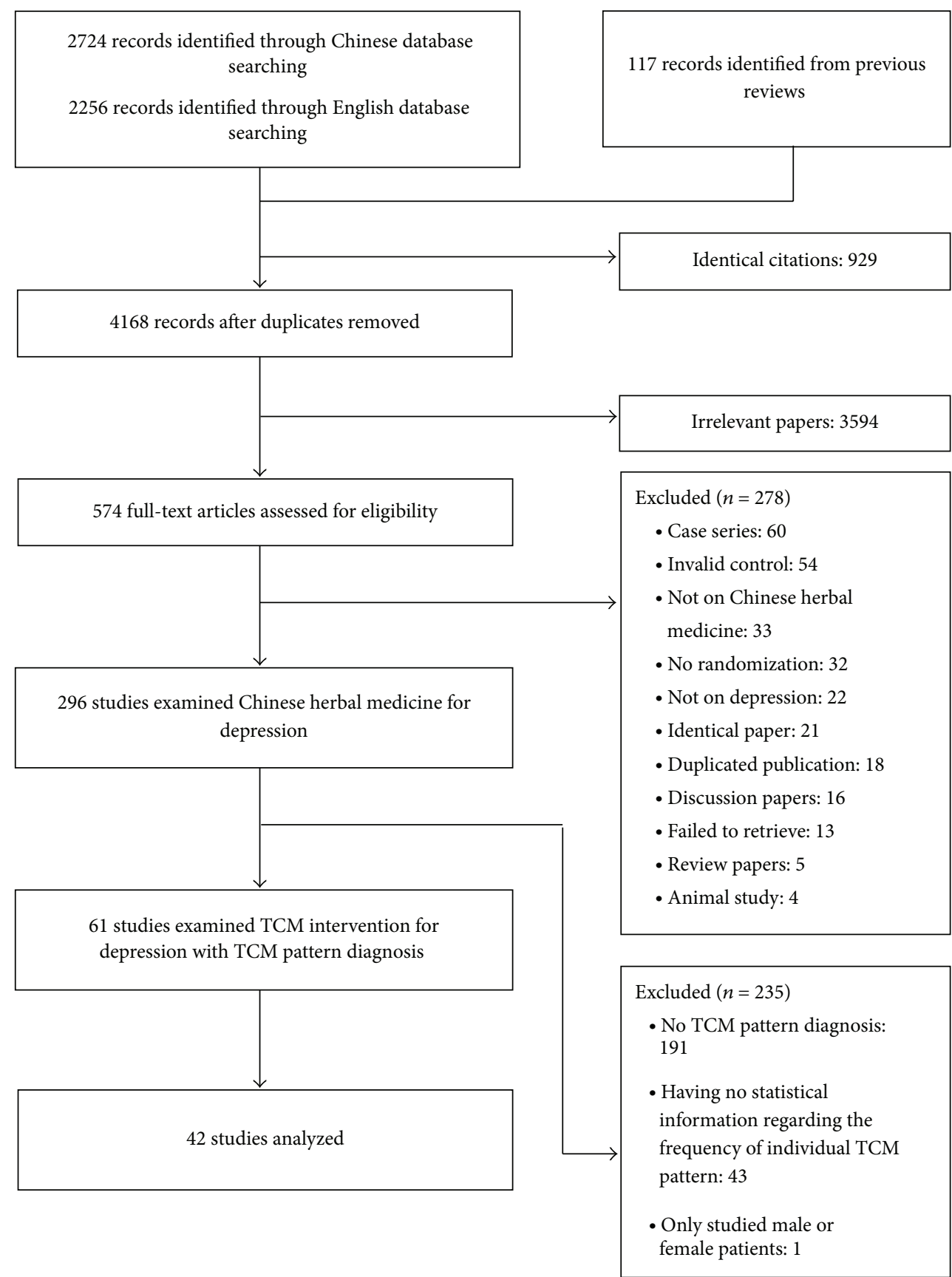

Figure 1: Study selection flowchart.

\section{Results}

The search yielded 5097 potential titles for review, of which 929 were duplicated records and 3594 were excluded for reasons of irrelevance. The full text of 574 was retrieved for detailed assessment, of which 278 were excluded for various reasons (Figure 1). Of the 296 studies on CHM for depression, 61 of them examined pattern-based treatment. A total of 27 different TCM patterns were identified in the 61 studies. We analyzed the most commonly studied
TCM patterns: liver qi depression, liver depression and spleen deficiency, dual deficiency of the heart and spleen, and liver depression and qi stagnation and liver-kidney yin deficiency. These four commonly studied TCM patterns were described in 42 of the 61 studies (68.9\%) and involved 1762 subjects accounting for $70.4 \%$ of the total 2504 subjects (Table 1 ). Eighteen of the 42 studies examined CHM alone and the other 24 studies examined CHM plus antidepressants [2465]. The 1762 participants had a mean age of 40.7 years, of which $59.0 \%$ were female. The participants were suffering 
TABLE 1: The most common TCM patterns diagnosed in people with depression.

\begin{tabular}{|c|c|c|c|}
\hline TCM pattern & Chinese name & $\begin{array}{l}\text { Number of subjects diagnosed with } \\
\text { the TCM pattern }(\%)(\text { total } N=1762)\end{array}$ & $\begin{array}{l}\text { Number of studies that examined the } \\
\text { TCM pattern }(\%)(\text { total } N=42)^{\mathrm{a}}\end{array}$ \\
\hline Liver qi depression & 肝氣穮結 & $797(45.2 \%)$ & $19(45.2 \%)$ \\
\hline Liver depression and spleen deficiency & 肝鬱脾虛 & $425(24.1 \%)$ & $13(31.0 \%)$ \\
\hline Dual deficiency of the heart and spleen & 心脾兩虛 & $315(17.9 \%)$ & $9(21.4 \%)$ \\
\hline Liver depression and qi stagnation & 肝鬱氣滯 & $225(12.8 \%)$ & $8(19.0 \%)$ \\
\hline
\end{tabular}

${ }^{a}$ Six studies examined more than one TCM pattern.

from depression unspecified in 33 of the 42 studies, six studies on poststroke depression and three on depression comorbid with diabetes. The diagnosis of depression was based on 17-item or 24-item Hamilton Depression Rating Scale $\left(\mathrm{HAMD}_{17 / 24}\right)$ in 36 studies, the Chinese Classification of Mental Disorder Second/Second-revised/Third Edition (CCMD-2/2-R/3) in 35 studies, Zung Self-rating Depression Scale (SDS) in seven studies, and one study each using DSMIV, Clinical Global Impression Scale (CGI), and International Classification of Diseases Version 10 (ICD-10). The response to intervention was assessed by the HAMD17/24 in 34 studies and by effective rate in 33 studies.

The criteria used for TCM pattern diagnosis were reported in 27 of the 42 studies. The criteria were based on the TCM Syndrome Diagnostic Standard $(N=7)$, New Guidelines for TCM Clinical Research $(N=3)$, TCM Diagnostic Standard for Depression $(N=2)$, Chinese Professional Association of Integrative Medicine Diagnostic Criteria for Mental Disorders, Version 1991/2001 ( $N=3$ ), Chinese Classification and Diagnostic Criteria of Mental Disorders $(N=1)$, and TCM textbooks $(N=13)$. However, none of the studies described other details of the diagnostic procedure and the background of practitioners who made the pattern diagnosis.

3.1. Methodological Quality. Twenty-three (54.8\%) of the 42 studies were described as randomized but the randomization method, blinding, and dropouts were not presented; hence, these 23 studies only had a Jadad scale score of one. Seventeen studies $(40.5 \%)$ had a Jadad scale score of two, and only two studies $(4.8 \%)$ obtained a Jadad scale score of four.

\subsection{Pattern-Based CHM Treatment}

3.2.1. Liver qi Depression. According to the TCM theory, liver qi depression is an impairment of the liver function, obstructing free movement of $q i$ and resulting in stagnation of $q i$ in liver [21]. Nineteen studies examined CHM treatment in patients with liver qi depression. Seven studies used CHM alone and 12 used CHM-antidepressant combination. Xiaoyao decoction was investigated in 13 (68.4\%) of the 19 studies, while other CHM formulas were studied in only one to two studies. Seventeen studies reported the ingredients of the CHM formulas for the treatment of liver qi depression. Chai Hu (Bupleurum chinense DC.) was the most commonly used herb, followed by Bai Shao (Paeonia lactiflora Pall.), Dang Gui (Angelica sinensis (Oliv.) Diels), Fu Ling (Poria Cocos (Schw) Wolf.), and Bai Zhu (Atractylodes macrocephala Koidz.). These five herbs were chosen for treating liver qi depression in more than half of the 17 studies that had reported the CHM ingredients (Table 2). The mean effective rate of Xiaoyao decoction for the treatment of liver qi depression was $84.7 \%$ and the mean HAMD change score was 19.1 (Table 3); for Xiaoyao decoction-antidepressant combination for liver qi depression, it was $86.0 \%$ and 19.1, respectively (Table 4). Tables 3 and 4 present the overall efficacy of pattern-based CHM monotherapy and CHM-antidepressant combination for liver qi depression.

3.2.2. Liver Depression and Spleen Deficiency. According to the TCM theory, liver depression and spleen deficiency is a pathological change in which the transporting and transforming function of the spleen is affected by depressed liver $q i$, leading to spleen deficiency [21]. Of the 13 studies on liver depression and spleen deficiency, three studies examined $\mathrm{CHM}$ alone and 10 investigated CHM-antidepressant combination. The most frequently used CHM formula was also Xiaoyao decoction, which was used in 9 of the 13 studies. Nine of the 13 studies provided ingredients of the CHM formulas. The commonly used single herbs for the treatment of liver depression and spleen deficiency were Bai Shao (Paeonia lactiflora Pall.), Fu Ling (Poria Cocos (Schw) Wolf.), Chai $\mathrm{Hu}$ (Bupleurum chinense DC.), Zhi Ke (Citrus $\times$ aurantium L.), Dang Gui (Angelica sinensis (Oliv.) Diels), and Bai Zhu (Atractylodes macrocephala Koidz.) (Table 2). Only one study was conducted on Xiaoyao decoction monotherapy for liver depression and spleen deficiency, and the HAMD change score was 7.3 (Table 3). The mean effective rate of Xiaoyao decoction-antidepressant combination for liver depression and spleen deficiency was $86.0 \%$ and the mean HAMD change score was 20.1 (Table 4). Tables 3 and 4 show the overall efficacy of pattern-based CHM monotherapy and CHMantidepressant combination for liver depression and spleen deficiency.

3.2.3. Dual Deficiency of the Heart and Spleen. Dual deficiency of the heart and spleen is a condition in which both heart blood and spleen qi are deficient, leading to disordered heart function and an impairment of the transporting and transforming function of spleen [21]. There was significant 


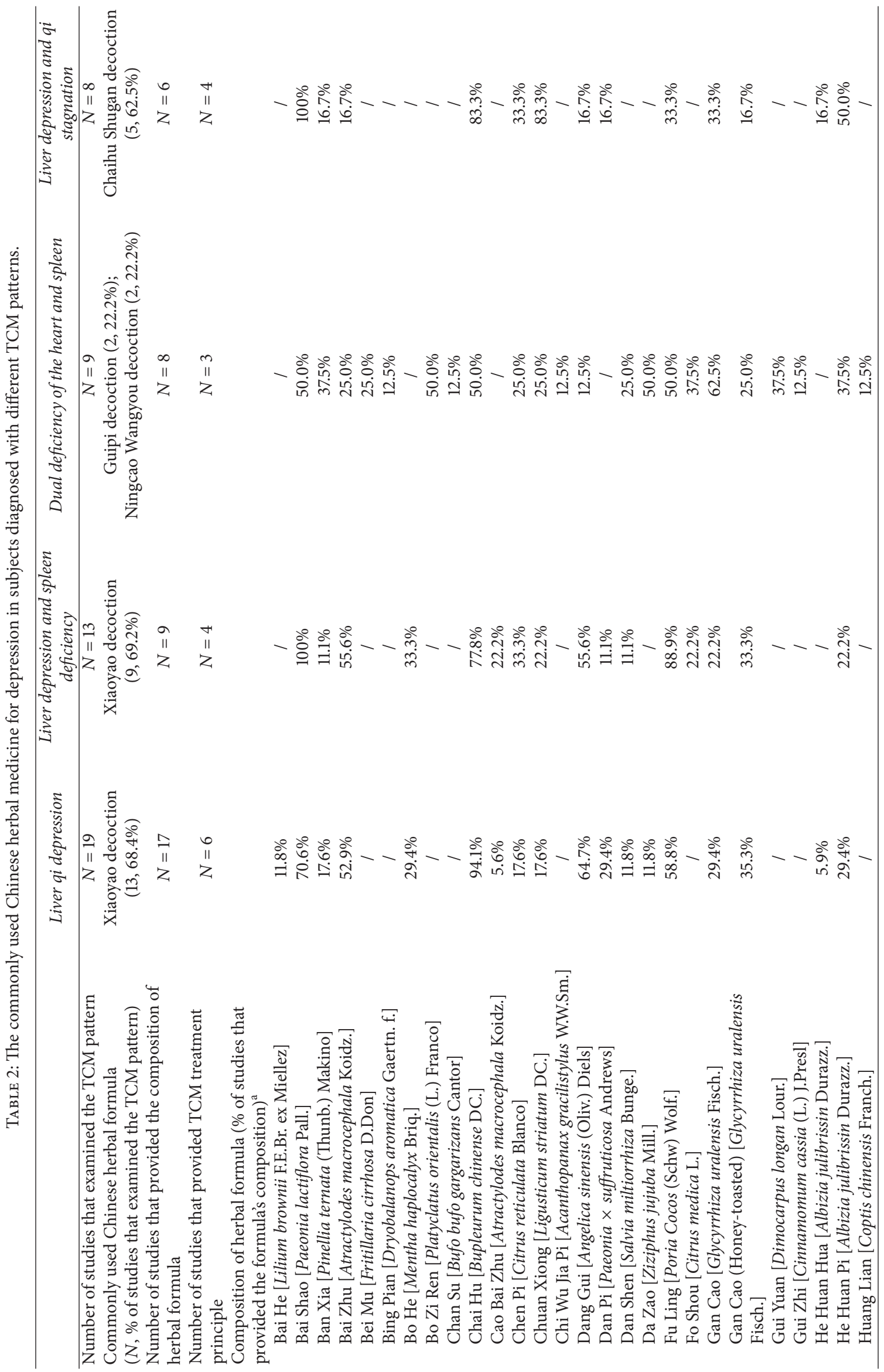




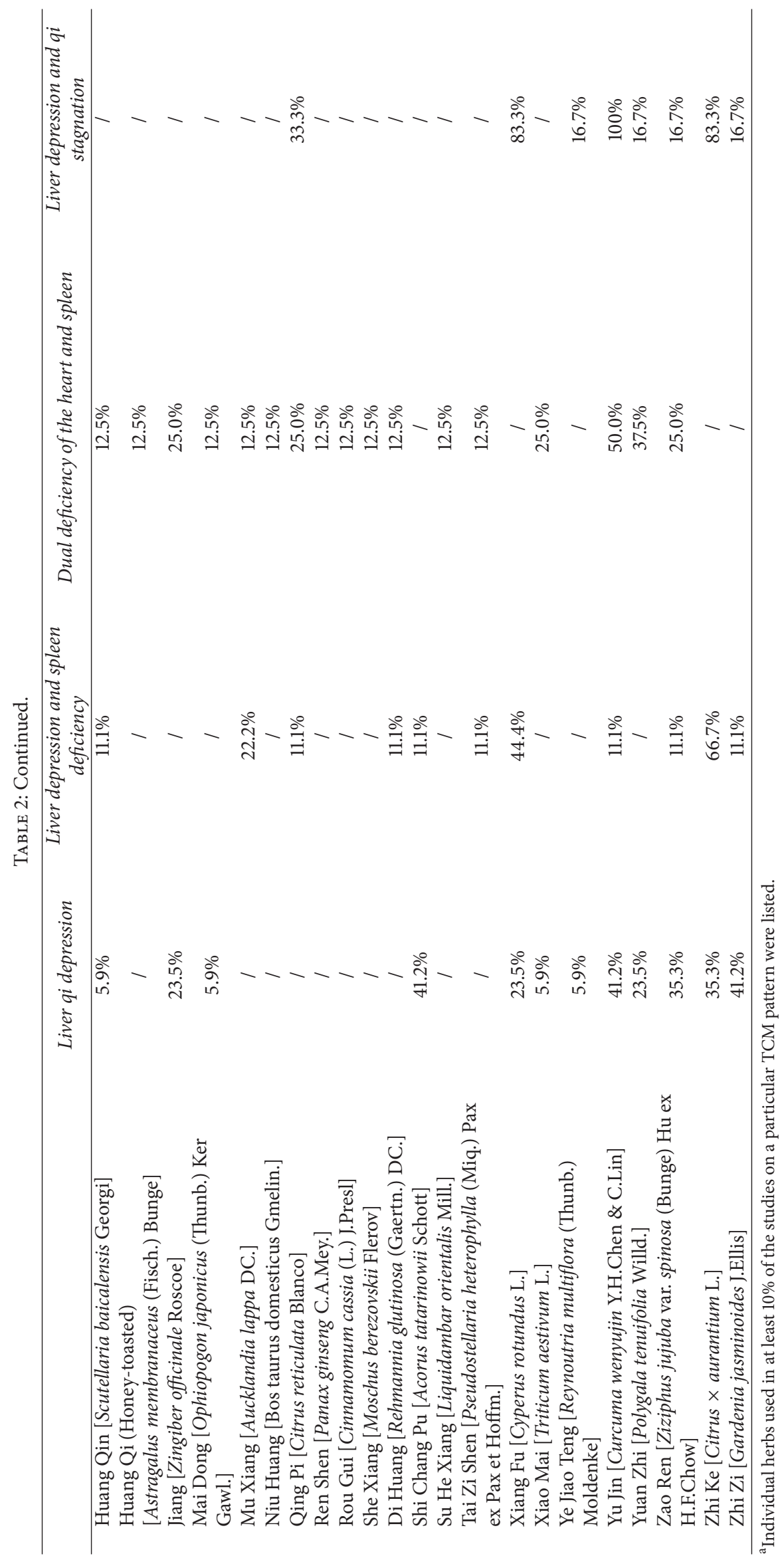




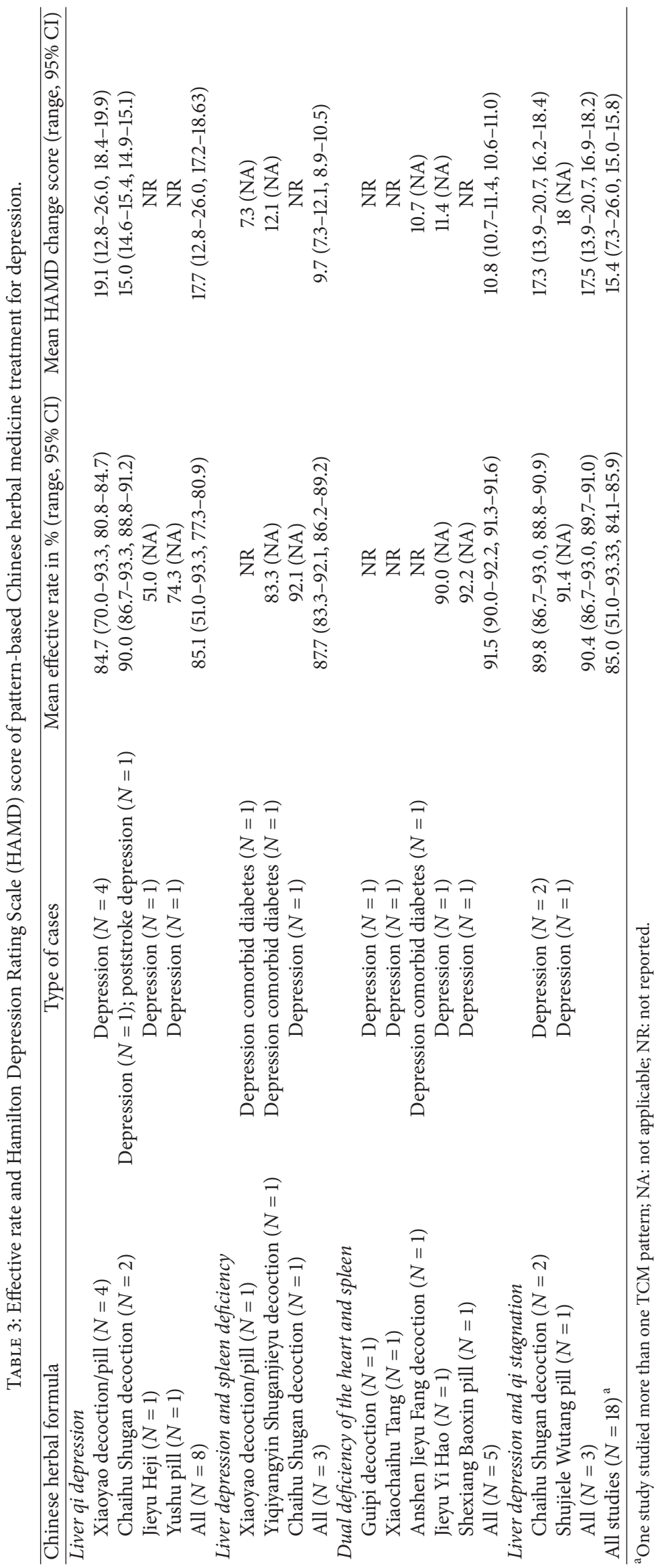




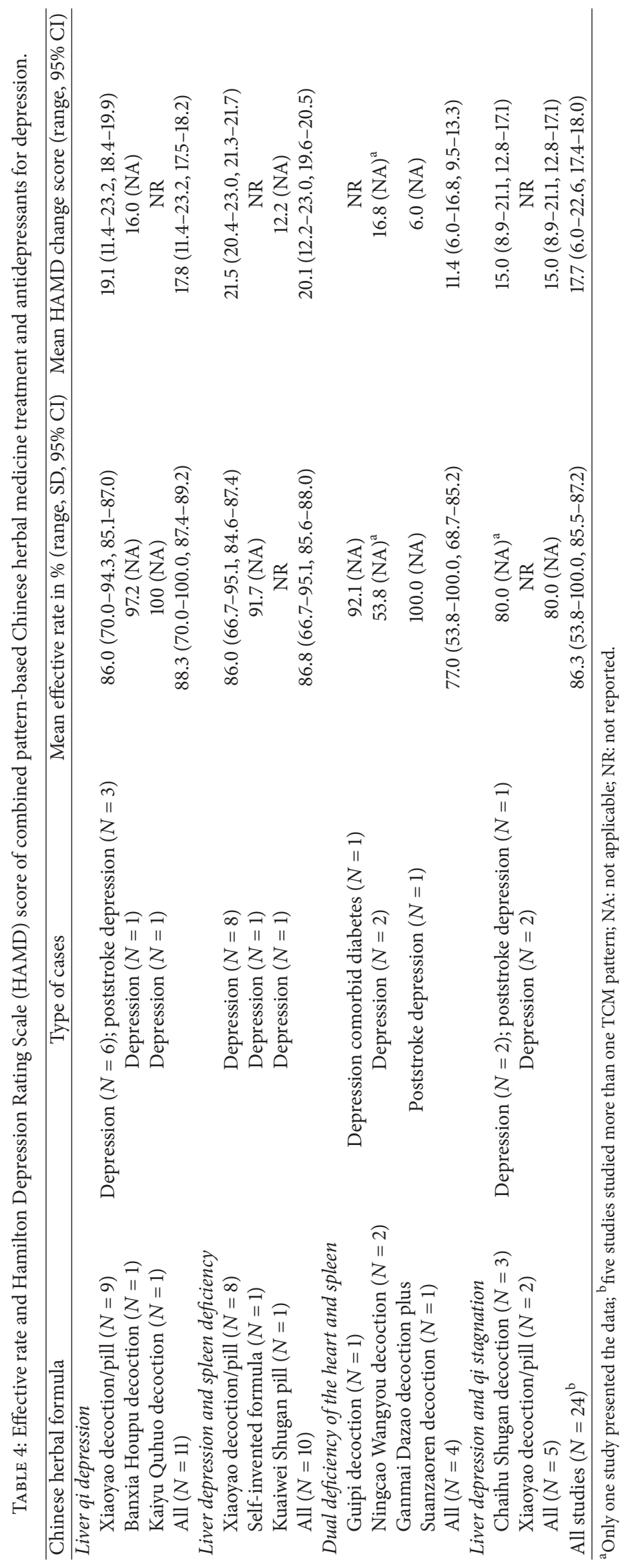


variation in the TCM pattern-based CHM treatment for dual deficiency of the heart and spleen. Of the 10 relevant studies, seven different CHM formulas were used. Guipi Tang and Ningcao Wangyou Tang were each used in two studies, while other CHM formulas were only examined once. The frequently used single herbs for dual deficiency of the heart and spleen were Gan Cao (Glycyrrhiza uralensis Fisch.), Bai Shao (Paeonia lactiflora Pall.), Chai Hu (Bupleurum chinense DC.), Fu Ling (Poria Cocos (Schw) Wolf.), Bo Zi Ren (Platyclatus orientalis (L.) Franco), Da Zao (Ziziphus jujua Mill.), and Yu Jin (Curcuma wenyujin Y. H. Chen \& C. Lin) (Table 2). The mean effective rate of CHM monotherapy for dual deficiency of the heart and spleen was $91.5 \%$ and the mean HAMD change score was 10.8 (Table 3); for CHM-antidepressant combination, it was $77.0 \%$ and 11.4 , respectively (Table 4 ).

3.2.4. Liver Depression and qi Stagnation. Liver depression and qi stagnation is a pathological change in which liver is depressed, leading to impeded circulation of $q i$ and stagnation of qi movement [21]. The most frequently used CHM formula for liver depression and qi stagnation was Chaihu Shugan decoction (Table 3). The commonly used single herbs were Bai Shao (Paeonia lactiflora Pall.), Yu Jin (Curcuma wenyujin Y. H. Chen \& C. Lin), Chai Hu (Bupleurum chinense DC.), Chuan Xiong (Ligusticum striatum DC.), Xiang Fu (Cyperus rotundus L.), Zhi Ke (Citrus $\times$ aurantium L.), Xiang $\mathrm{Fu}$ (Cyperus rotundus L.), and He Huan Pi (Albizia julibrissin Durazz.) (Table 2). The mean effective rate of CHM monotherapy for liver depression and qi stagnation was $90.4 \%$ and the mean HDRS change score was 17.5 (Table 3); for CHM-antidepressant combination, it was $80.0 \%$ and 15.0 , respectively (Table 4 ).

\section{Discussion}

This study is the first systematic review of English and Chinese literature, involving 61 studies and 2504 subjects on the classification and treatment of depression using the TCM diagnostic system. We found that the TCM pattern diagnoses for depression were diverse. Among 27 different TCM patterns identified, liver qi depression was the most commonly diagnosed TCM pattern in people with depression, followed by liver depression and spleen deficiency, dual deficiency of the heart and spleen, and liver depression and qi stagnation. With regard to CHM treatment, Xiaoyao decoction was the most frequently used herbal formula for the treatment of liver qi depression and liver depression and spleen deficiency, while Chaihu Shugan decoction was often used for liver depression and qi stagnation. For dual deficiency of the heart and spleen, no single formula could be regarded as commonly used across TCM practitioners. The results suggest that TCM practitioners may be more consistent in the treatment of depression involving liver depression than other patterns. The abundance of low-quality studies highlights that knowledge and experience in conducting high-quality RCTs may be limited. It further suggests that institutional review boards and publishing journals should play an active role in monitoring the standards of clinical trials.
The present paper found that Bai Shao (Paeonia lactiflora Pall.), which has a function of nourishing the blood and emolliating the liver, and Chai $\mathrm{Hu}$ (Bupleurum chinense DC.), which can sooth the liver, were commonly used to treat depression regardless of the TCM pattern. Animal studies have found that extract from Bai Shao produces antidepressant effects in chronic unpredictable stress-induced depression model in mice and rats [66]. The antidepressant effects are likely mediated by inhibition of the monoamine oxidase activity and oxidative stress, upregulation of neurotrophins, and modulation of the function of the hypothalamic-pituitary-adrenal axis [66]. Pharmacological studies of Chai $\mathrm{Hu}$ have shown that it has hepatoprotective, anti-inflammatory, antipyretic, analgesic, and immunomodulatory effects [67-70]; however, its antidepressant effects remain unclear. According to a TCM textbook, Chai $\mathrm{Hu}$ is a "Sovereign" herb in Xiaoyao decoction and Chaihu Shugan decoction [69]. The "Sovereign" herb is used for treating the principal diseases based on the TCM theory; the "Minister" herb has synergistic effects with "Sovereign" herbs and helps to alleviate other accompanying symptoms; the "Assistant" herb is for enhancing the therapeutic effects and modulating the adverse effects of "Sovereign" and "Minister" herbs, while the "Courier" herb is used for harmonizing the actions of the others [66]. Therefore, some of the commonly used herbs identified in this review may not primarily be targeted at depression; instead, they indirectly alleviate depression by enhancing or harmonizing the actions of other herbs. In view of the common use of Chai Hu in the treatment of depression, further studies on its antidepressant effects are warranted.

Xiang Fu (Cyperus rotundus L.) and He Huan Pi (Albizia julibrissin Durazz.) were specifically used for liver depression and qi stagnation, and Bo Zi Ren (Platyclatus orientalis (L.) Franco), which has a function of nourishing the heart and tranquilizing shen, was specific for dual deficiency of the heart and spleen. Yu Jin (Curcuma wenyujin Y. H. Chen \& C. Lin) was commonly used for liver depression and $q i$ stagnation and dual deficiency of the heart and spleen, Fu Ling (Poria Cocos (Schw) Wolf.) for liver qi depression, liver depression and spleen deficiency, and dual deficiency of the heart and spleen, and Dang Gui (Angelica sinensis (Oliv.) Diels) and Bai Zhu (Atractylodes macrocephala Koidz.) for liver qi depression and liver depression and spleen deficiency. Since three of the four commonly diagnosed TCM patterns in people with depression involve liver depression and two involve spleen deficiency, the prescription of Chinese herbs for different TCM patterns are inevitably overlapping. A lack of consistency across TCM practitioners in their selection of herbal formulas and pattern-based prescription of individual herbs may also lead to variation in CHM treatment [70]. Considering the limited number of studies available, further research on pattern-based CHM treatment for depression is warranted.

Due to the studies' variation in study design and inadequate methodological quality, it is difficult to conclude which herbal formulas have higher efficacy and which TCM patterns respond better to $\mathrm{CHM}$. As a whole, there is no evidence to suggest CHM-antidepressant combination has higher efficacy than CHM monotherapy for depression. We found 
that the effective rate was generally high for pattern-based treatment in liver qi depression and was similar between CHM monotherapy and CHM-antidepressant combination. For liver depression and spleen deficiency, the efficacy of CHM monotherapy and CHM-antidepressant combination was similar in terms of effective rate, but it was lower with CHM monotherapy in terms of mean HAMD change score (9.7 versus 20.1 for CHM-antidepressants combination). For dual deficiency of the heart and spleen, the efficacy of pattern-based CHM treatment was relatively weaker, especially CHM-antidepressant combination, which had a mean effective rate of $77.0 \%$ and mean HAMD change score of 11.4. For liver depression and qi stagnation, the efficacy of CHM monotherapy and CHM-antidepressant combination was similar, except for a relatively low mean effective rate of CHM-antidepressant combination $(80.9 \%$ versus $90.4 \%$ for CHM monotherapy). It is clear that further studies with better methodological quality are needed to delineate the efficacy of pattern-based CHM treatment in depression.

There are some methodologic limitations of the study. First, there is no "gold standard" in the classification of TCM patterns, and the criteria for each pattern might be different between researchers. Future studies using both Western and Chinese medicine systems in diagnosis and severity assessment may facilitate Western-Chinese medicine integration in the understanding and treatment of depression. Although we reported the pattern diagnosis by the authors, the procedure and quality of the diagnostic process was uncertain. Such uncertainties would inevitably lead to discrepancies in the selection of herbs in treatment. Although a large number of studies were reviewed, this paper only summarized the effective rate and HAMD change score based on RCTs; metaanalysis was not possible due to difference in study design and low methodological quality of the studies.

Despite the limitations, the present study, for the first time, systematically and comprehensively summarized important data on pattern-based CHM treatment for depression. Our data should be useful for both clinical practice and future research. More high quality studies incorporating TCM pattern differentiation and treatment principle are needed to examine the efficacy of TCM treatments and the additional benefit of pattern differentiation.

\section{Conclusion}

We found that liver qi depression, liver depression and spleen deficiency, dual deficiency of the heart and spleen, and liver depression and qi stagnation were the most commonly studied TCM patterns in people with depression. In addition, Bai Shao (Paeonia lactiflora Pall.) and Chai $\mathrm{Hu}$ (Bupleurum chinense DC.) were commonly used across different TCM patterns regardless of the prescribed Chinese herbal formulas. Due to the limited number of studies on TCM patternbased treatment of depression and their low methodological quality, we are unable to draw any conclusion regarding which herbal formulas have higher efficacy and which TCM patterns respond better to CHM.

\section{Conflict of Interests}

The authors have no conflict of interests to report.

\section{Acknowledgment}

The study was supported by the Chinese Medicine Research Fund of the Hospital Authority of Hong Kong (Project no. HACMR001).

\section{References}

[1] A. J. Ferrari, F. J. Charlson, R. E. Norman et al., "Burden of depressive disorders by country, sex, age, and year: findings from the global burden of disease study 2010," PLoS Medicine, vol. 10, no. 11, Article ID e1001547, 2013.

[2] American Psychiatric Association, Diagnostic and Statistical Manual of Mental Disorders, American Psychiatric Association, Washington, DC, USA, 4th edition, 1994.

[3] E. Bromet, L. H. Andrade, I. Hwang et al., "Cross-national epidemiology of DSM-IV major depressive episode," BMC Medicine, vol. 9, no. 1, article 90, 2011.

[4] R. C. Kessler, J. Soukup, R. B. Davis et al., "The use of complementary and alternative therapies to treat anxiety and depression in the United States," The American Journal of Psychiatry, vol. 158, no. 2, pp. 289-294, 2001.

[5] M. C. Hsu, D. Creedy, W. Moyle, L. Venturato, S. L. Tsay, and W. C. Ouyang, "Use of complementary and alternative medicine among adult patients for depression in Taiwan," Journal of Affective Disorders, vol. 111, no. 2-3, pp. 360-365, 2008.

[6] N. A. Qureshi and A. Mohammed, "Mood disorders and complementary and alternative medicine: a literature review," Neuropsychiatric Disease and Treatment, vol. 9, pp. 639-658, 2013.

[7] F. Qin, X.-A. Wu, Y. Tang, Q. Huang, Z.-J. Zhang, and J.-H. Yuan, "Meta-analysis of randomized controlled trials to assess the effectiveness and safety of free and easy Wanderer plus, a polyherbal preparation for depressive disorders," Journal of Psychiatric Research, vol. 45, no. 11, pp. 1518-1524, 2011.

[8] H. Zhao, X. Wan, and J.-X. Chen, "A mini review of traditional Chinese medicine for the treatment of depression in China," The American Journal of Chinese Medicine, vol. 37, no. 2, pp. 207-213, 2009.

[9] L. Butler and K. Pilkington, "Chinese herbal medicine and depression: the research evidence," Evidence-Based Complementary and Alternative Medicine, vol. 2013, Article ID 739716, 14 pages, 2013.

[10] M. J. Kou and J. X. Chen, "Integrated traditional and Western medicine for treatment of depression based on syndrome differentiation: a meta-analysis of randomized controlled trials based on the Hamilton depression scale," Journal of Traditional Chinese Medicine, vol. 32, no. 1, pp. 1-5, 2012.

[11] W.-F. Yeung, K.-F. Chung, K.-Y. Ng, Y.-M. Yu, E. Tat-Chi Ziea, and B. Fung-Leung Ng, "A meta-analysis of the efficacy and safety of traditional Chinese medicine formula Ganmai Dazao decoction for depression," Journal of Ethnopharmacology, vol. 153, no. 2, pp. 309-317, 2014.

[12] X. K. Qing, P. Li, M. Han et al., "Systematic review of randomize controlled trials of Xiaoyao powder in treatment of depression," Journal of Traditional Chinese Medicine, vol. 51, no. 6, pp. 500$505,2010$. 
[13] Y. Wang, R. Fan, and X. Huang, "Meta-analysis of the clinical effectiveness of traditional Chinese medicine formula ChaihuShugan-San in depression," Journal of Ethnopharmacology, vol. 141, no. 2, pp. 571-577, 2012.

[14] J. Liu, Y. Zhang, M. Han, Z. Liu, J. Wang, and Q. He, “Chinese herbal formula Xiao Yao San for treatment of depression: a systematic review of randomized controlled trials," EvidenceBased Complementary and Alternative Medicine, vol. 2012, Article ID 931636, 13 pages, 2012.

[15] W. Yang, F. Meng, and Y. Jiang, Diagnostics of Traditional Chinese Medicine, Academy Press, Beijing, China, 1998.

[16] R. N. Schnyer and B. Flaws, Curing Depression Naturally with Chinese Medicine, Blue Poppy Press, Boulder, Colo, USA, 1998.

[17] Y. Wang, M. Li, X. Dai, and G. Fan, Internal Chinese Medicine, Shanghai Scientific and Technological Documentation Publishing House, Shanghai, China, 1997.

[18] A. Bensoussan, N. J. Talley, M. Hing, R. Menzies, A. Guo, and M. Ngu, "Treatment of irritable bowel syndrome with Chinese herbal medicine: a randomized controlled trial," Journal of the American Medical Association, vol. 280, no. 18, pp. 1585-1589, 1998.

[19] Q.-L. Zha, Y.-T. He, and J.-P. Yu, "Correlations between diagnostic information and therapeutic efficacy in rheumatoid arthritis analyzed with decision tree model," Chinese Journal of Integrated Traditional and Western Medicine, vol. 26, no. 10, pp. 871-876, 2006.

[20] W.-F. Yeung, K.-F. Chung, K.-Y. Ng, Y.-M. Yu, E. T.-C. Ziea, and B. F.-L. Ng, "A systematic review on the efficacy, safety and types of Chinese herbal medicine for depression," Journal of Psychiatric Research, vol. 57, no. 7, pp. 165-175, 2014.

[21] World Health Organization, WHO International Standard Terminologies on Traditional Medicine in the Western Pacific Region, World Health Organization, Western Pacific Region, 2007.

[22] B. Peng and J. Xie, Traditional Chinese Internal Medicine, People's Medical Publishing House, Beijing, China, 2nd edition, 2007.

[23] A. R. Jadad, R. A. Moore, D. Carroll et al., "Assessing the quality of reports of randomized clinical trials: is blinding necessary?" Controlled Clinical Trials, vol. 17, no. 1, pp. 1-12, 1996.

[24] B. Lin and J. Xia, "Clinical observation on modified chaihu shugan san on depression," Journal of New Chinese Medicine, vol. 4, no. 8, pp. 36-37, 2011 (Chinese).

[25] B. Y. Yan, "Clinical observation on integrated traditional Chinese and Western medicine in treating depression," Guangming Journal of Chinese Medicine, vol. 24, no. 9, pp. 1761-1762, 2009 (Chinese).

[26] B. Zhou and H. Yan, "Efficacy of integrated traditional Chinese and Western medicine on 30 cases of depression caused by liver stagnation and spleen deficiency," Tianjin Journal of Traditional Chinese Medicine, vol. 29, no. 4, pp. 329-331, 2012 (Chinese).

[27] D. L. Zhaou, J. Xia, J. Li, and Y. Y. Ma, "Clinical observation on treatment of depressive disorder by fluvoxamine combined with Xiaoyao pills," Sichuan Mental Health, vol. 25, no. 2, pp. 97-99, 2012 (Chinese).

[28] E. P. Xu, "Treatment of danzhi xiaoyao san combining fluoxetine in treating 70 cases of post-stroke depression," Research of Chinese Medicine, vol. 19, no. 4, pp. 31-32, 2006 (Chinese).

[29] F. Mou and X. Y. Zhou, "Clinical observation of Shexiang baoxin pills in treating 129 cases of depression," Chinese Traditional Patent Medicine, vol. 26, no. s1, pp. 69-70, 2004 (Chinese).
[30] F. Zhang, "Xiaoyao San treatment of depression 60 cases," Journal of Practical Traditional Chinese Internal Medicine, vol. 26, no. 6, pp. 37-38, 2012 (Chinese).

[31] G.-H. Yu, S.-C. Liang, and Q.-Z. Sun, "Study on modified xiaoyao decoction combining clomipamine in treating depression," Chinese Journal of Integrated Traditional and Chinese Medicine, vol. 27, no. 4, pp. 318-320, 2007 (Chinese).

[32] H. D. Zhang and H. Su, "Clinical comparative study of paroxetine combined with Xiaoyao pill of depression," Modern Journal of Integrated Traditional Chinese and Western Medicine, vol. 18, no. 33, pp. 4060-4063, 2009 (Chinese).

[33] H. Li, S. Xu, B. Li et al., "Xiaoyao San combining fluoxetine in treating 41 cases of depression caused by liver depression and spleen deficiency," Shaanxi Journal of Traditional Chinese Medicine, vol. 30, no. 1, pp. 49-50, 2009 (Chinese).

[34] H. M. Wang and Q. H. Dong, "Clinical observation of anseng jieyu fang in treating depression in patients with type 2 diabetes," Journal of New Chinese Medicine, vol. 44, no. 10, pp. 72-73, 2012 (Chinese).

[35] H. Y. Wang, B. J. Zhang, X. Liu, and Y. D. Tao, "Clinical observation on treatment of first-episode depression by duloxetine combined with Xiaoyaowan mixture," Sichuan Mental Health, vol. 26, no. 1, pp. 32-34, 2013 (Chinese).

[36] H. Zhang and S. H. Li, "Kaiyu quhuo tang combining fluoxetine capsule in treating 44 cases of depression," Shaanxi Journal of Traaditional Chinese Medicine, vol. 33, no. 6, pp. 674-675, 2012 (Chinese).

[37] J. Huo, H. P. Fu, J. Yang et al., "The clinical research of depressive disorder treated in dan-zhi decoction for ease combined fluoxetine," Journal of Henan University of Chinese Medicine, vol. 23, no. 136, pp. 38-39, 2010 (Chinese).

[38] J. P. Xian, Q. Zhou, and X. D. Li, "Clinical observation of modified xiaoyao san combining paroxetine in treating depression," Contemporary Medicine, vol. 16, no. 13, pp. 154-155, 2010 (Chinese).

[39] J. R. Zhang, "Effect of xiao chaihu tang in treating 40 cases of depression," Beijing Journal of TCM, vol. 22, no. 5, pp. 38-39, 2003 (Chinese).

[40] J. Y. Zheng, "Observation on the efficacy of Ganmai Dazao Tang combining Suan Zaoren Tang in treating post-stroke depression," Beijing Journal of Traditional Chinese Medicine, vol. 28, no. 4, pp. 291-292, 2009.

[41] J. Z. Zhang, "Efficacy analysis on integrated traditional Chinese and Western medicine in treating 64 cases of depression," Chinese Journal of Practical Nervous Diseases, vol. 15, no. 10, pp. 39-40, 2012 (Chinese).

[42] K. Cheng, H. Yan, and K. J. Duan, "Clinical observation on the efficacy of self-invented Chinese medicine in treating depression," Clinical Journal of Traditional Chinese Medicine, vol. 20, no. 4, pp. 375-376, 2008 (Chinese).

[43] L. J. Mao, Y. Lu, and W. X. Song, "Observation on the efficacy of yushu pills in treating 70 cases of depression," Chinese Journal of Integrative Medicine on Cardio-cerebrovascular Disease, vol. 4, no. 12, pp. 1047-1048, 2006 (Chinese).

[44] L. R. Li, Z. X. Chen, and L. Kong, "Jieyu heji on 50 cases of depression," Guanming Journal of Chinese Medicine, vol. 25, no. 12, pp. 2206-2207, 2010 (Chinese).

[45] M. Zhang and W. Z. Xiao, "Clinical observation on Danzhi Xiaoyao powder in treating depression in patients with diabetes," Journal of Liaoning University of TCM, vol. 10, no. 2, pp. 108-109, 2008 (Chinese). 
[46] P. Zhou, L. Q. Chen, X. M. Peng et al., "Clinical observation on banxia houpu decoction combining fluoxetine in treating depressive adolescents," Chinese Journal of Integrative Medicine on Cardio-cerebrovascular Disease, vol. 9, no. 2, pp. 247-248, 2011 (Chinese).

[47] R. C. Wang, "Clinical observation on modified xiaoyao san in treating depression," Medical Journal of Chinese Peoples Health, vol. 20, no. 13, pp. 1504-1505, 2008 (Chinese).

[48] R. X. Yang, Y. Dong, and D. M. Wang, "Clinical observation on Shujiele Watang pills in treating depression," Chinese Medicine Focus, vol. 1, no. 5, pp. 16-17, 2002 (Chinese).

[49] S. Y. Liu, "Observation of chaihushugan powder plus psychological nursing intervention therapy on acute poststroke depression," Journal of Emergency in Traditional Chinese Medicine, vol. 8, no. 8, pp. 1205-1207, 2012 (Chinese).

[50] W. F. Long and Z. H. Dan, "Jieyu Yi Hao in treating 30 cases of depression caused by dual deficiency of the heart and spleen," Journal of New Chinese Medicine, vol. 43, no. 3, pp. 38-39, 2010 (Chinese).

[51] W. X. Huang, Y. H. Yin, and J. J. Hu, "Chaihu Shugan powder combining deanxit on 32 cases of post-stroke depression," Fujian Journal of Traditional Chinese Medicine, vol. 41, no. 5, pp. 21-22, 2010 (Chinese).

[52] X. Gao, "Xiaoyao powder combined with escitalopram oxalate tablets in the treatment of Depression," China Modern Doctor, vol. 50, no. 1, pp. 66-70, 2012 (Chinese).

[53] Y. C. Ma, Z. X. Chen, W. D. Jin et al., "Preliminary observation on treating depression in the integrative medicine," Clinical Journal of Chinese Medicine, vol. 3, no. 1, pp. 6-7, 2011 (Chinese).

[54] Y. F. Ma, "Study on modified xiaoyao san combining fluoxetine in treating 80 cases of post-stroke," Journal of Noah China Coal Medical University, vol. 12, no. 2, pp. 187-188, 2010 (Chinese).

[55] Y. J. Li, H. C. Luo, and R. Q. Qian, "Effect of danzhi xiaoyao san on depressive patients' neuro -immune-endocrine system," Chinese Journal of Integrative Medicine, vol. 27, no. 3, pp. 197200, 2007 (Chinese).

[56] Y. K. Huang, J. Chen, and Y. Q. Huang, "Clinical observation on the treatment efficacy of Xiayao pills on 62 cases of depression caused by liver qi depression," Fujian Journal of Traditional Chinese Medicine, vol. 40, no. 5, pp. 3-4, 2009 (Chinese).

[57] Y. L. Liu and S. L. Dong, "Observation on the efficacy of Danzhi Xiaoyao San in treating depression in patients with hypertension," Chinese Journal of Integrated Traditional and Chinese Medicine, vol. 28, no. 3, pp. 280-281, 2008 (Chinese).

[58] Y. M. Guo, C. F. Liu, and J. J. Yang, "Chinese medicine on 38 cases of depression," China's Naturopathy, vol. 9, no. 10, pp. 5657, 2001 (Chinese).

[59] Y. M. Song, "Treating 30 cases of depression using the method of Shugan Jieyu liqi," Chinese Medicine Modern Education of China, vol. 9, no. 16, pp. 7-8, 2011 (Chinese).

[60] Y. Wang and S. S. Liu, "Clinical observation on danzhi xiaoyao san combining fluoxetine in treating depression," Journal of Community Medicine, vol. 5, no. 11, pp. 57-58, 2007 (Chinese).

[61] Y. Q. Jian, "Pattern-based integrated traditional Chinese and Western medicine treatment on 30 cases of depression," Liaoning Journal of Traditional Chinese Medicine, vol. 28, p. 236, 2001 (Chinese).

[62] Y. X. Wang, Y. B. Gao, H. Zhou, Z. Guan, and X. Z. Shang, "Qi-Yin nourishing method to treat diabetes with depression," World Journal of Traditional Chinese Medicine, vol. 5, no. 6, pp. 397-399, 2010 (Chinese).
[63] Z. Lian and Q. Wu, "Treatment efficacy analysis of chaihu shugan powder combining fluoxetine in treating post-stroke depression," Guangming Journal of Chinese Medicine, vol. 24, no. 1, pp. 101-102, 2009 (Chinese).

[64] Z. X. Bao, Q. Tian, W. Zhou, W. Sun, and G. P. He, "Clinical observation of influence of Zhuyang Shuxin recipe on TCM syndrome of patients with depression with syndrome of liver and kidney yang deficiency," Chinese Archives of Traditional Chinese Medicine, vol. 30, no. 10, pp. 116-118, 2012 (Chinese).

[65] Z. X. Chen, Y. C. Ma, W. D. Jin et al., "Clinical study on the treatment of depressive patients caused by different TCM pattern using integrated traditional Chinese and Western medicine," International Journal of Traditional Chinese Medicine, vol. 33, no. 5, pp. 421-424, 2011 (Chinese).

[66] Q.-Q. Mao, S.-P. Ip, Y.-F. Xian, Z. Hu, and C.-T. Che, "Antidepressant-like effect of peony: a mini-review," Pharmaceutical Biology, vol. 50, no. 1, pp. 72-77, 2012.

[67] H.-X. Sun, "Haemolytic activities and adjuvant effect of Bupleurum chinense saponins on the immune responses to ovalbumin in mice," Vaccine, vol. 24, no. 9, pp. 1324-1331, 2006.

[68] D. Xie, X. Jia, B. Cai, and L. Zhang, "Experimental study on anti-inflammatory and analgesic effects of volatile oil of Bupleurum chinense and B. scorzonerifolium," Asian Journal of Pharmaceutical and Clinical Research, vol. 15, pp. 108-110, 2007.

[69] Y. Yang, Chinese Medical Formulae, Beijing Shi: Ren min wei sheng chu ban she, Beijing, China, 2001, (Chinese).

[70] W.-F. Yeung, K.-F. Chung, M. M.-K. Poon et al., "Prescription of Chinese herbal medicine and selection of acupoints in patternbased traditional Chinese medicine treatment for insomnia: a systematic review," Evidence-based Complementary and Alternative Medicine, vol. 2012, Article ID 902578, 16 pages, 2012. 


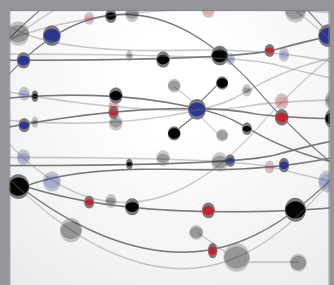

The Scientific World Journal
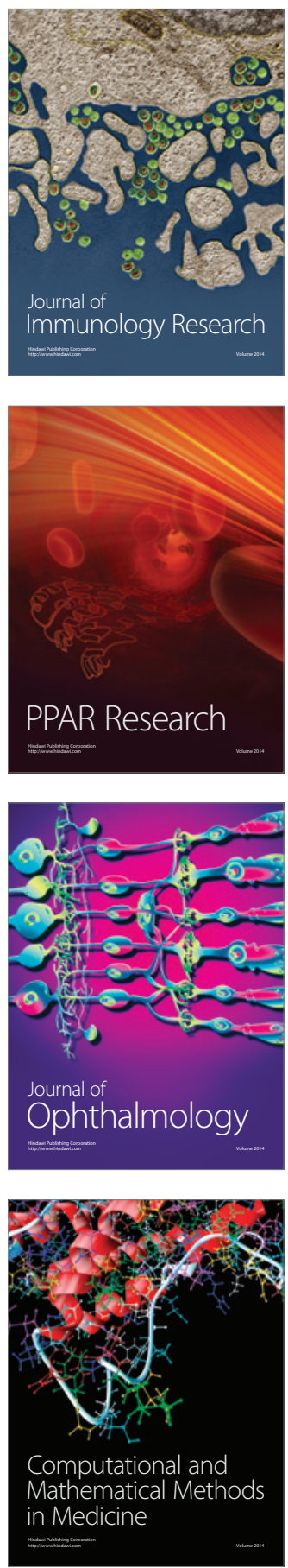

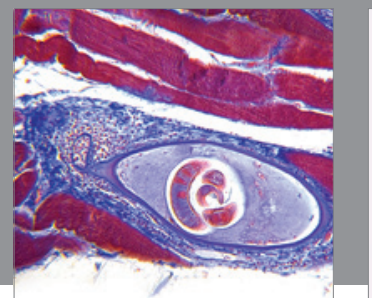

Gastroenterology

Research and Practice
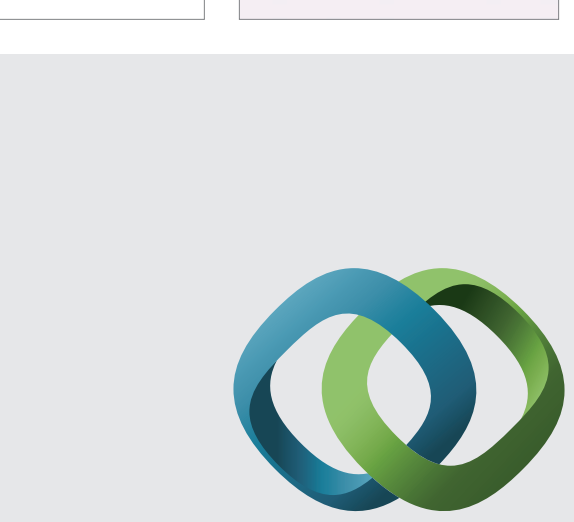

\section{Hindawi}

Submit your manuscripts at

http://www.hindawi.com
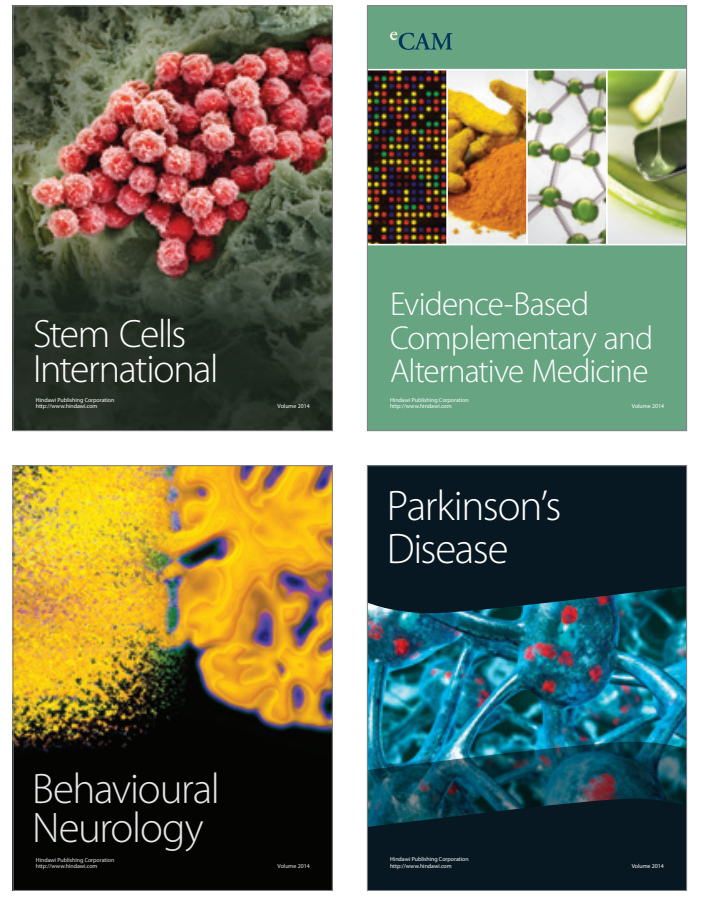
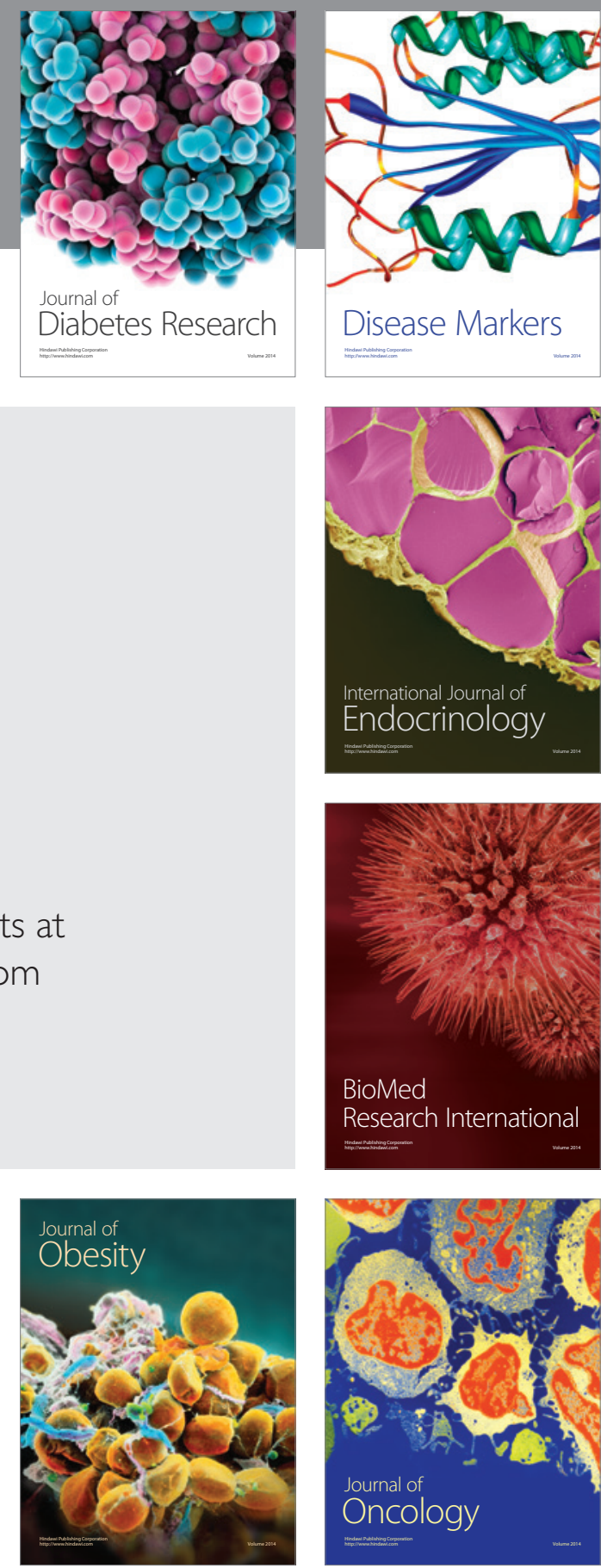

Disease Markers
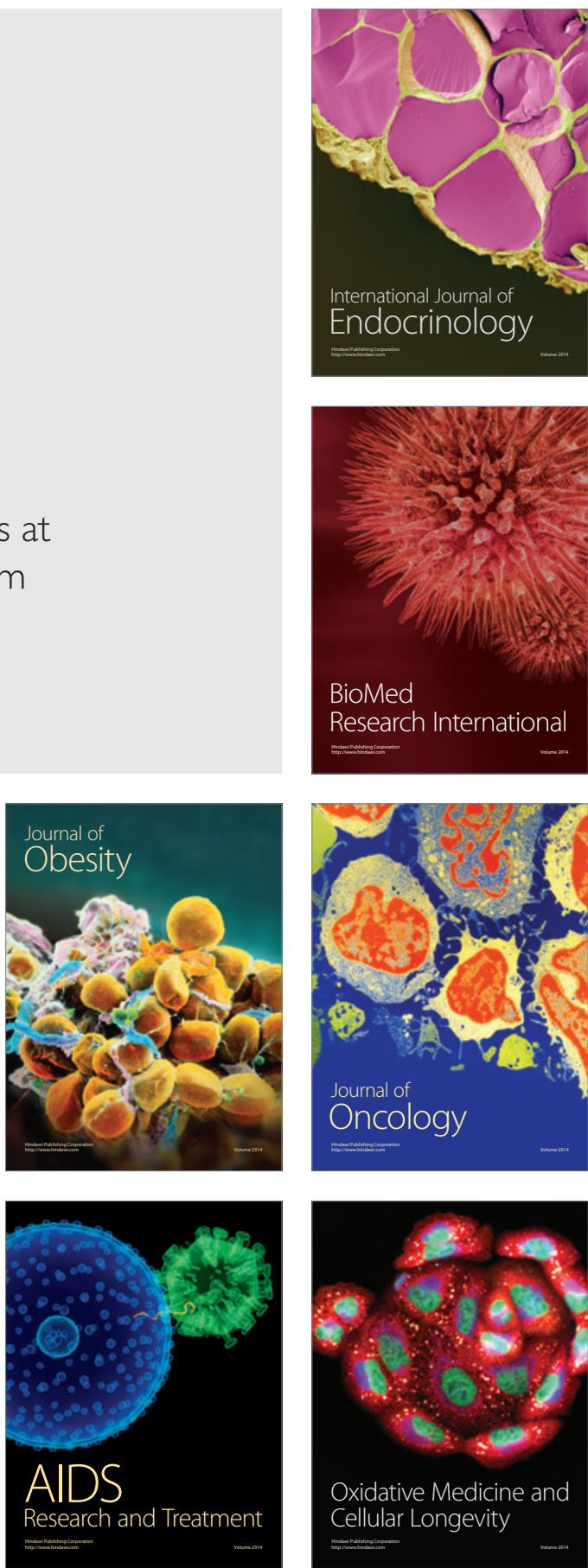